\title{
Fault Detection of a Blending Tank Process Using Mixed Integer Linear Programming
}

\author{
Soheil Salehpour
}

Thomas Gustafsson and Andreas Johansson

The northernmost University of Technology in Scandinavia World-class research and education 


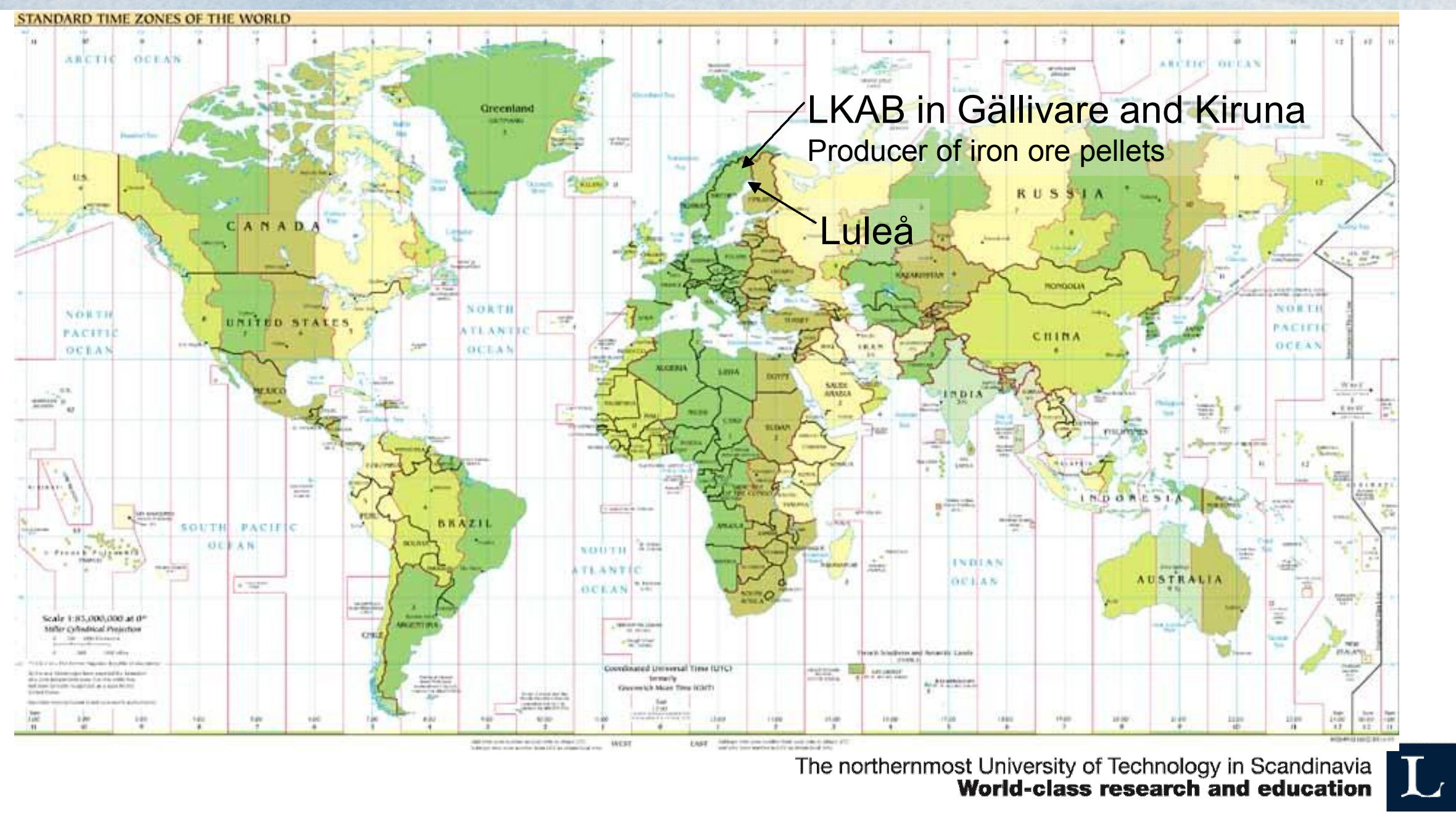

IFACMMM 2009 Workshop on Automation in Mining, Mineral and Metal Industry

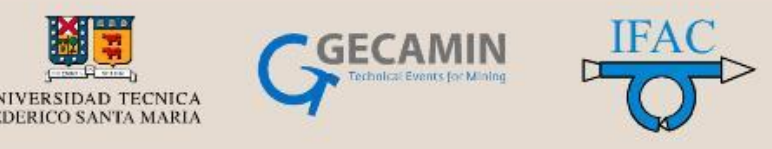




\section{Why Fault Detection?}

The northernmost University of Technology in Scandinavia World-class research and education 


\section{Flow Diagram from LKAB Malmberget and Kiruna Ore Pellet Process}


$\rightarrow$ KIRUNA PELLETIZING PLANT KK3

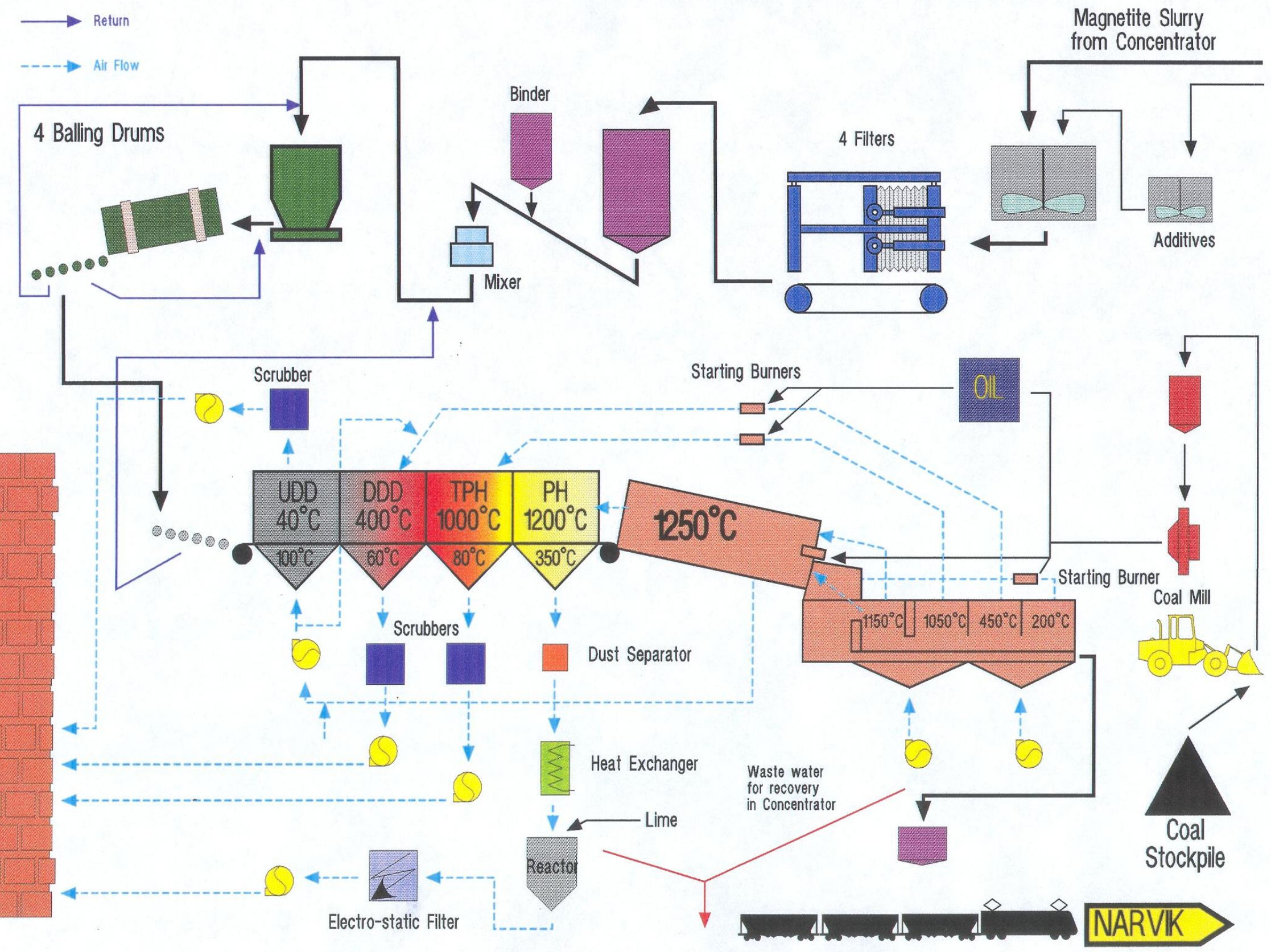




\section{Iron Ore Pellet Process}

-The refinement from rock to pellets is called pelletization process and includes the following main steps:

- Drilling, blasting and initial crushing of the rock.

- Magnetic separators and crushers separate the iron ore from the tailings.

- Further refinement is achieved by grinding and cleaning of the iron ore.

- The resulting slurry is then mixed with a binding media (bentonite).

- Balling in drums.

- The green pellets are finally dried and sintered in a pelletizing machine 


\section{Flow diagram of Ore Pellet Process}

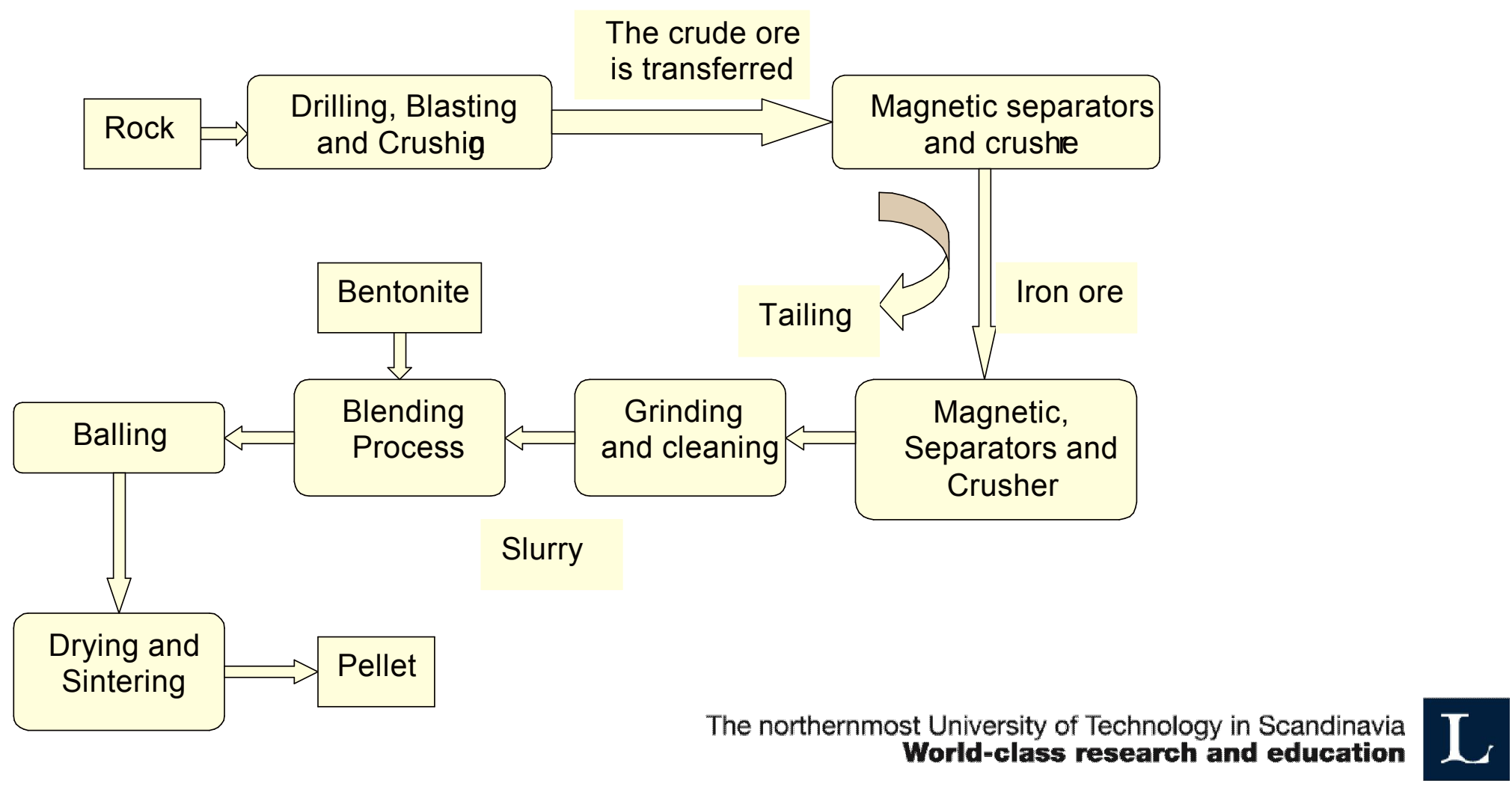

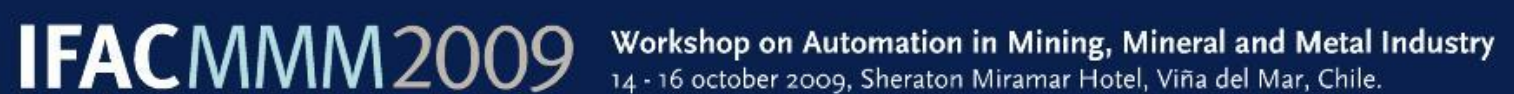

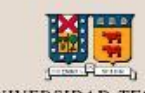




\section{Bentonite Blending Process}

- The quality of iron ore pellets depends on many factors.

- One important issue is the mixing of binding material and slurry.

- The proper mixing is achieved by keeping the material in the blender tank at an appropriate level to achieve a suitable residence time. 
-Inflows: Slurry, Returned oversize, Bentonite.

-Measured: Mass inside the tank.

-PI-controller: Control the level of the blender by regulating the blender's hinged outflow-valve.

The modelling of the hinged valve is important.

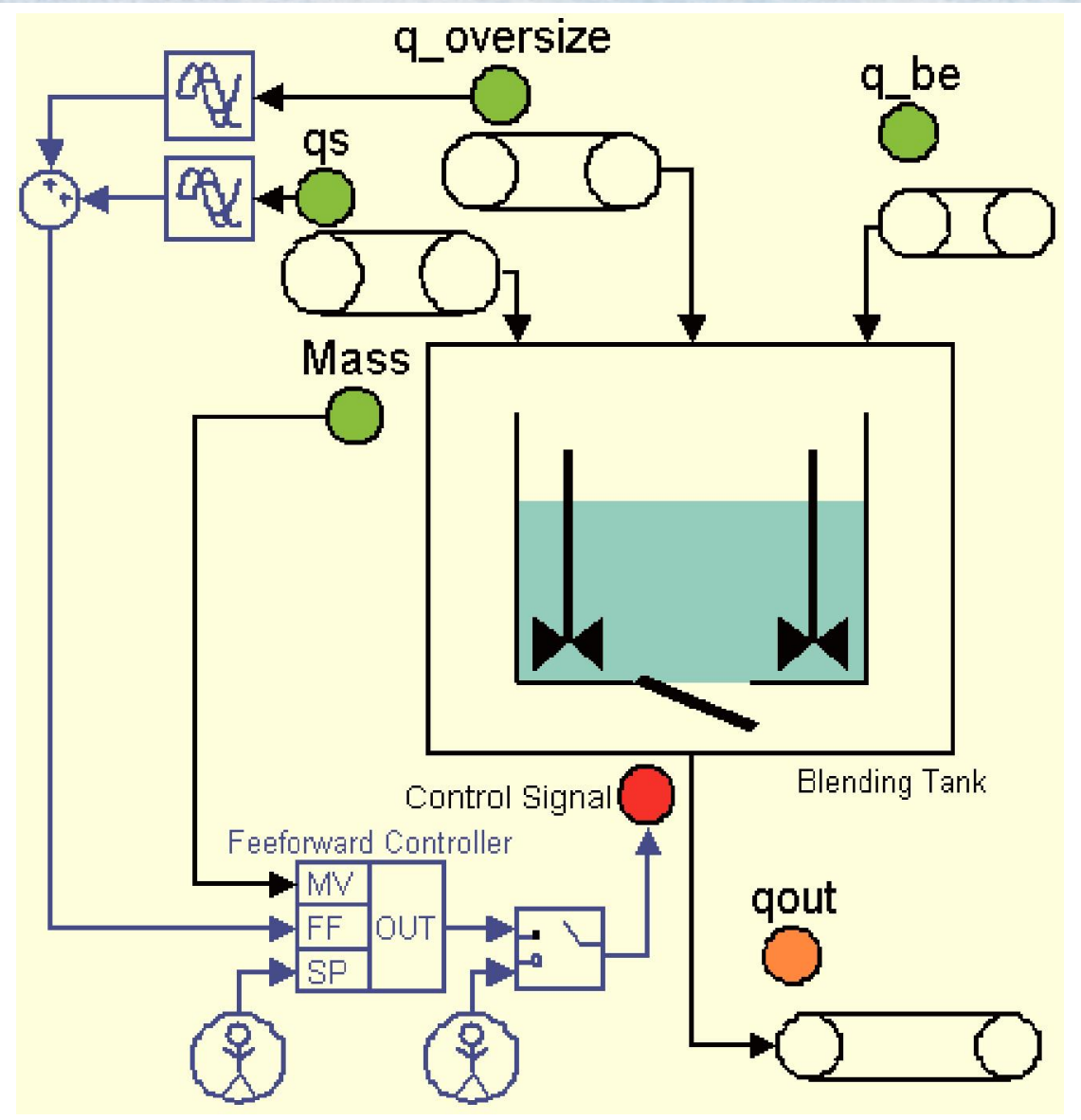

The northernmost University of Technology in Scandinavia World-class research and education

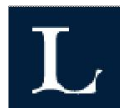




\section{Two detrimental conditions}

1) The hinged valve is coated with slurry and therefore has to be cleaned to maintain its function.

2) Slurry is improperly distributed so that it does not cover the outflow valve, which then loses its authority over outflow. 


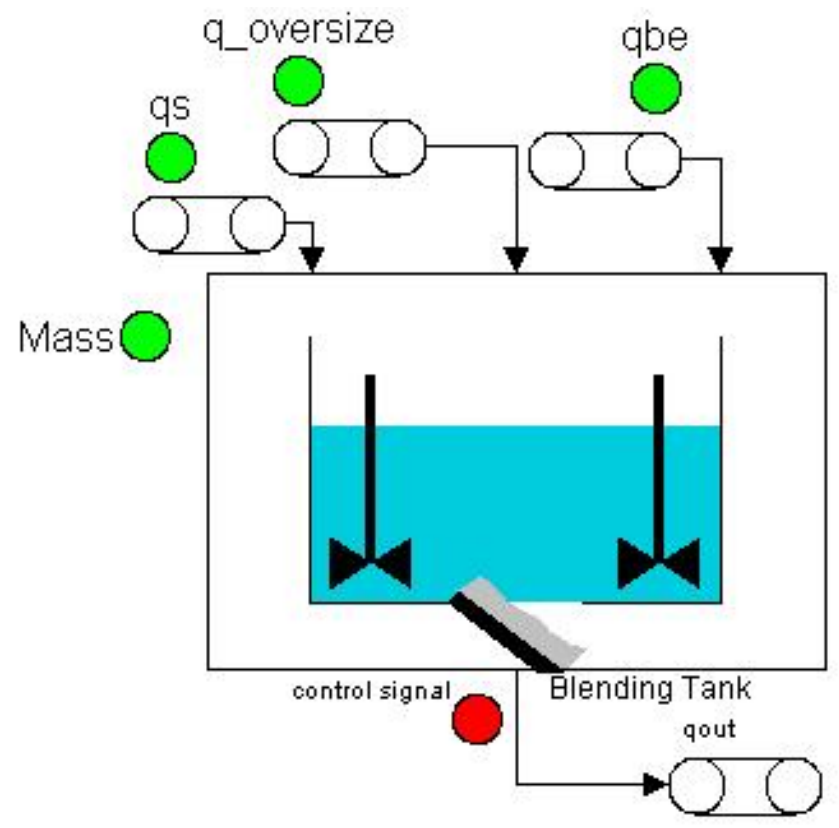

- Bentonite blending process whenever the hinged valve is coated.

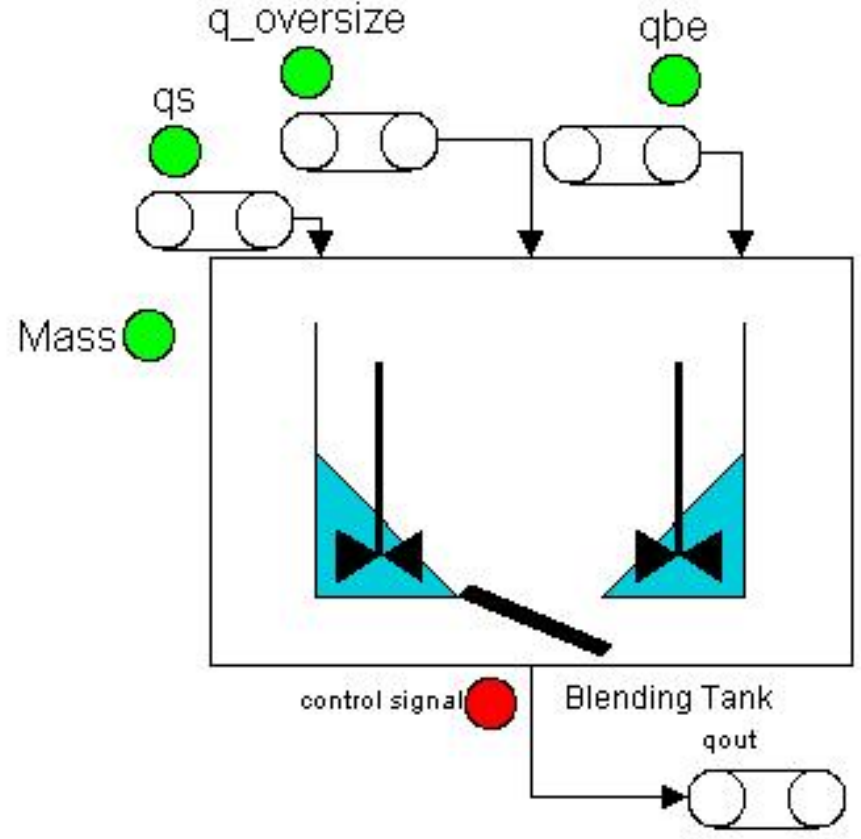

- Bentonite blending process in the absence of slurry around the hinged valve at low levels

The northernmost University of Technology in Scandinavia World-class research and education 


\section{Process Model}

-The flow through the hinged valve can be expressed as

$$
\mathrm{q}_{\text {out }}=\left(\theta_{0}+\theta_{1} \mathrm{u}(\mathrm{t})\right) \sqrt{2 \mathrm{gh}(\mathrm{t})}
$$

-Where $h(t)$ is the level of the tank and $u(t)$ is the control signal. The process model is as

-Then

$$
\mathrm{q}_{\text {out }}(\mathrm{t})=\mathrm{q}_{\text {in }}(\mathrm{t})-\dot{\mathrm{m}}(\mathrm{t})
$$

$$
\int_{t_{0}}^{t_{1}} q_{\text {in }}(\tau) d \tau-m\left(t_{1}\right)+m\left(t_{0}\right)=\int_{t_{0}}^{t_{1}} q_{\text {out }}(\tau) d \tau=\left[\int_{t_{0}}^{t_{1}} \sqrt{2 \operatorname{gh}(\tau)} d \tau, \int_{t_{0}}^{t_{1}} u(\tau) \sqrt{2 \operatorname{gh}(\tau)} d \tau\right]\left[\theta_{0}, \theta_{1}\right]^{T}
$$

$\theta_{0}$ and $\theta_{1}$ change when the valve gets coated. 


\section{Nonlinear Behaviour of the hinged outflow-valve}

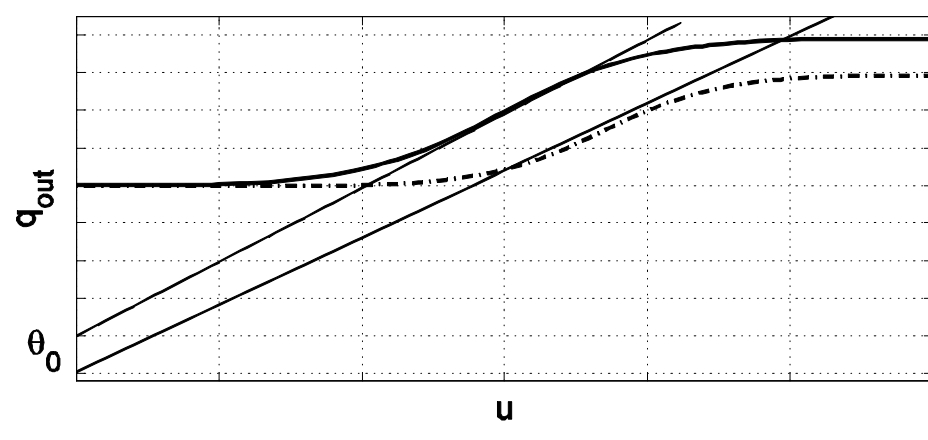

(a)

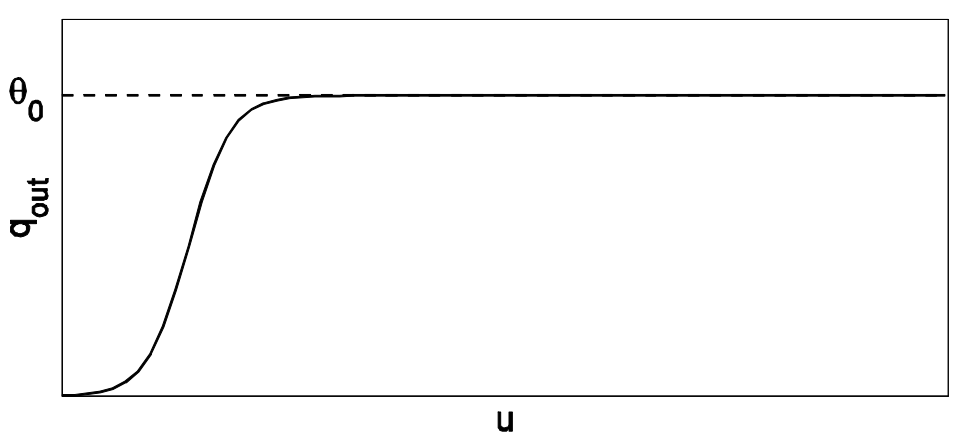

(b)

(a) Nominal characteristic and linearization of it (solid) and the characteristic of the coated valve (dash-dotted)

(b) Characteristic when the slurry does not cover the valve 


\section{Method Based on MILP and the Derivative of Uncertainty}


We present a method based on the assumption of piecewise constant parameters which results in a sparse structure of their derivative.

A MILP (Mixed Integer Linear Programming) algorithm to maximize the sparsity of a matrix is introduced.

The northernmost University of Technology in Scandinavia World-class research and education 
LULEA UNIVERSITY OF TECHNOLOGY

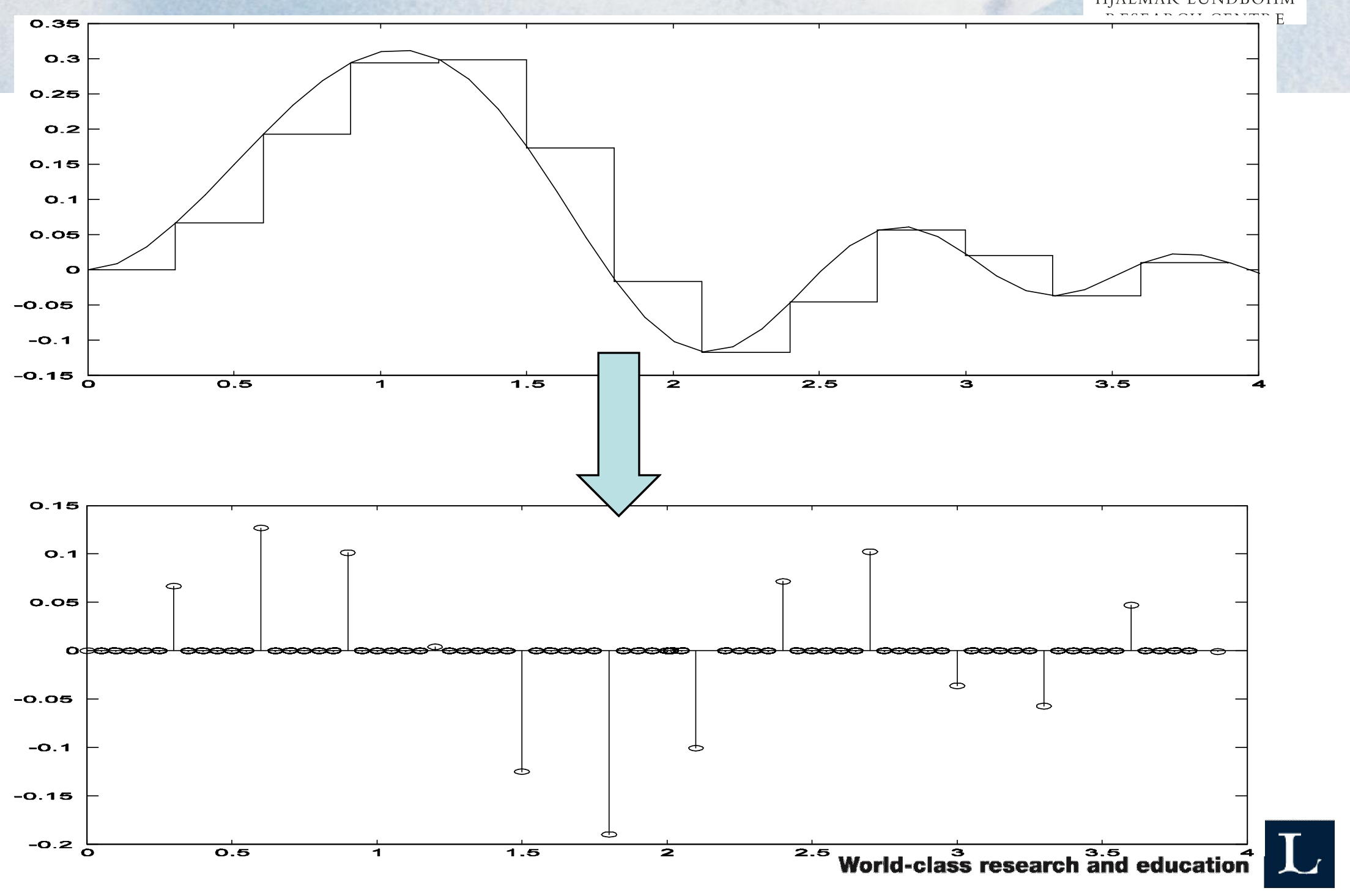

IFACMMM 2009 Workshop on Automation in Mining, Mineral and Metal Industry

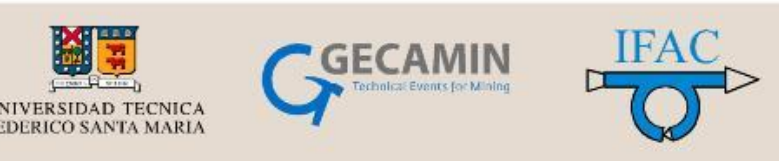




\section{SPARSE MATRIX}

-A sparse matrix is a matrix populated primarily with zeros.

-An exact solution of sparsity by using MILP (Mixed Integer Linear Programming) to minimize the number of non-zero elements in a matrix or vector.

-We consider the logical variable $\delta_{\mathrm{ij}} \in\{0,1\}$ and the matrix $S=\left(s_{i j}\right)_{i, j=1, \ldots, N}$ then:

$$
\begin{aligned}
& \min \text { imize } \sum_{\mathrm{i}, \mathrm{j}=1}^{\mathrm{N}} \delta_{\mathrm{ij}} \\
& \text { subject to : }-\mathrm{M} \delta_{\mathrm{ij}} \leq \mathrm{s}_{\mathrm{ij}} \leq \mathrm{M} \delta_{\mathrm{ij}}
\end{aligned}
$$




\section{DISTURBANCE BOUND}

The moving window norm gives a bounded measure of the disturbance

$$
\left\|v_{\infty}\right\|=\sup _{t \geq 0} \sum_{i=0}^{k} \omega(k-i)|v(i)|
$$

Where $\omega(k)$ is monotone decreasing for $k>0$.

An assumption on the disturbance $v=\left[v_{1}, \ldots, v_{1}\right]$ may now be expressed as

$$
\left\|v_{j}\right\|_{\infty} \leq \varepsilon_{j}
$$

the condition (1) can be expressed as

-

-where

$$
\mathrm{W}=\left(\begin{array}{ccc}
\omega(0) & \cdots & 0 \\
\vdots & \ddots & \vdots \\
\omega(\mathrm{N}) & \cdots & \omega(0)
\end{array}\right) \quad \omega=\operatorname{diag}\left(\omega_{1}, \ldots, \omega_{1}\right) \quad \varepsilon=\operatorname{diag}\left(\varepsilon_{1}, \ldots, \varepsilon_{1}\right)
$$




\section{Time-varying process model}

-A time-varying linear regression model

$$
y(t)=\varphi(t)^{T} \theta(t)+e(t)
$$

where $\theta(t)$ is a piecewise constant vector and $e(t)$ is some noise signal. For an ARX $\left(n_{a}, n_{b}, n_{c}\right)$ model

$$
\begin{aligned}
& \phi(t)^{\mathrm{T}}=\left[-\mathrm{y}_{\mathrm{t}-1}, \ldots,-\mathrm{y}_{\mathrm{t}-\mathrm{n}_{\mathrm{a}}}, \mathrm{u}_{\mathrm{t}-\mathrm{n}_{\mathrm{k}}}, \ldots, \mathrm{u}_{\mathrm{t}-\mathrm{n}_{\mathrm{k}}-\mathrm{n}_{\mathrm{b}}+1}\right] \\
& \theta(\mathrm{t})^{\mathrm{T}}=\left[\mathrm{a}_{\mathrm{t}}^{1}, \mathrm{a}_{\mathrm{t}}^{2}, \ldots, \mathrm{a}_{\mathrm{t}}^{\mathrm{n}_{\mathrm{s}}}, \mathrm{b}_{\mathrm{t}}^{1}, \mathrm{~b}_{\mathrm{t}}^{2}, \ldots, \mathrm{b}_{\mathrm{t}}^{\mathrm{n}_{\mathrm{t}}}\right]
\end{aligned}
$$




\title{
Fault Detection Based on MILP and the Derivative of Parameters
}

\author{
$\max \underset{\Pi}{\operatorname{mimize}}(\operatorname{sparsity}$ of $\Delta \Theta)-\mathrm{H \varepsilon}$ \\ subject to: $W\left|Y_{m}-\Xi \Theta\right| \leq \varepsilon$
}

where $\quad \mathrm{Y}_{\mathrm{m}}=\left[\mathrm{y}_{\mathrm{m}}(1), \ldots, \mathrm{y}_{\mathrm{m}}(\mathrm{N})\right]^{\mathrm{T}}, \Xi=\operatorname{diag}\left(\phi(1)^{\mathrm{T}}, \ldots, \phi(\mathrm{N})^{\mathrm{T}}\right)$ 


\section{Simulation Result}
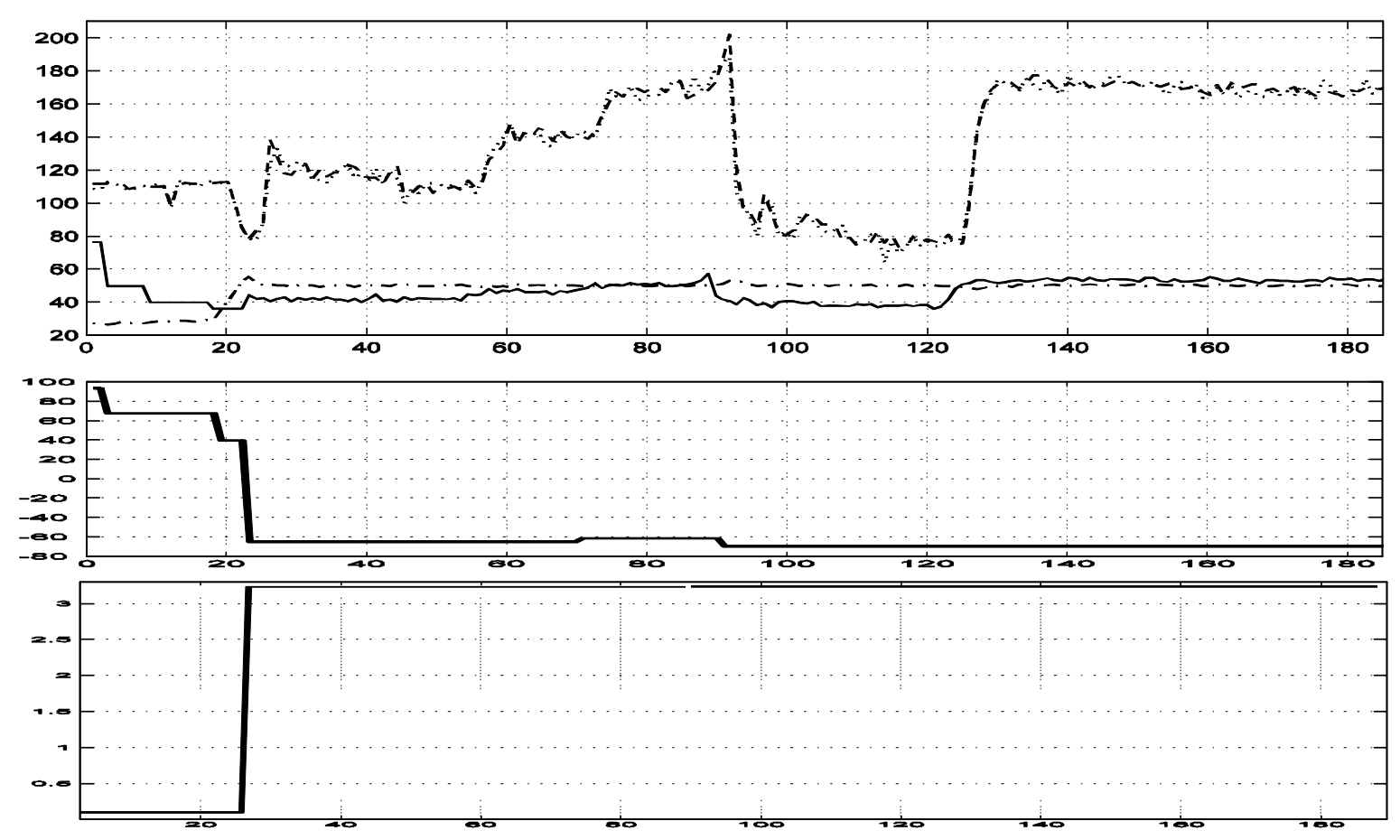

- The hinged valve model change detection (a) The control signal (solid), the level of the tank (dash-dotted), the computed outflow, the estimated outflow (dashed), $\begin{array}{lll}\text { (b) Estimated } \boldsymbol{\theta}_{0} & \text { (c) Estimated } \boldsymbol{\theta}_{1} \quad \begin{array}{c}\text { The northernmost University of Technology in Scandinavia } \\ \text { World-class research and education }\end{array}\end{array}$ 


\section{Contributions and Future Work}

-Present efficient methods for fault detection and model quality estimation.

- Demonstrate the method for Blending process.

- The disadvantage of this method is the computational complexity of the MILP-optimization.

-Demonstrate and evaluate our methods for use in robust control.

-Find some convex problem description to decrease the computational complexity.

-The "LASSO approach" maybe a good choice.

The northernmost University of Technology in Scandinavia World-class research and education 


\section{Thanks!}

The northernmost University of Technology in Scandinavia World-class research and education

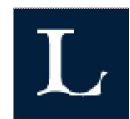

